\title{
RESEARCH
}

\section{Hotspot DAXX, PTCH2 and CYFIP2 mutations in pancreatic neuroendocrine neoplasms}

\author{
T Vandamme1,2,*, M Beyens1,*, G Boons', A Schepers³, K Kamp², K Biermann4, P Pauwels5, W W De Herder², \\ L J Hofland ${ }^{2}$, M Peeters' ${ }^{1}$, G Van Camp ${ }^{3}$ and K Op de Beeck ${ }^{1}$ \\ ${ }^{1}$ Center of Oncological Research (CORE), University of Antwerp, Antwerp, Belgium \\ 2Section of Endocrinology, Department of Internal Medicine, Section of Endocrinology, Erasmus Medical Center, Rotterdam, The Netherlands \\ ${ }^{3}$ Center of Medical Genetics, University of Antwerp, Antwerp, Belgium \\ ${ }^{4}$ Department of Pathology, Erasmus Medical Center, Rotterdam, The Netherlands \\ ${ }^{5}$ Department of Pathology, University of Antwerp, Antwerp, Belgium
}

Correspondence should be addressed to T Vandamme: timon.vandamme@uantwerpen.be

*(T Vandamme and M Beyens contributed equally to this work)

\begin{abstract}
Mutations in DAXX/ATRX, MEN1 and genes involved in the phosphoinositide-3-kinase/ Akt/mammalian target of rapamycin (PI3K/Akt/mTOR) pathway have been implicated in pancreatic neuroendocrine neoplasms (pNENs). However, mainly mutations present in the majority of tumor cells have been identified, while proliferation-driving mutations could be present only in small fractions of the tumor. This study aims to identify high- and low-abundance mutations in pNENs using ultra-deep targeted resequencing. Formalinfixed paraffin-embedded matched tumor-normal tissue of 38 well-differentiated pNENs was sequenced using a HaloPlex targeted resequencing panel. Novel amplicon-based algorithms were used to identify both single nucleotide variants (SNVs) and insertiondeletions (indels) present in $>10 \%$ of reads (high abundance) and in $<10 \%$ of reads (low abundance). Found variants were validated by Sanger sequencing. Sequencing resulted in 416,711,794 reads with an average target base coverage of $2663 \pm 1476$. Across all samples, 32 high-abundance somatic, 3 germline and 30 low-abundance mutations were withheld after filtering and validation. Overall, $92 \%$ of high-abundance and $84 \%$ of low-abundance mutations were predicted to be protein damaging. Frequently, mutated genes were MEN1, DAXX, ATRX, TSC2, PI3K/Akt/mTOR and MAPK-ERK pathway-related genes. Additionally, recurrent alterations on the same genomic position, so-called hotspot mutations, were found in DAXX, PTCH2 and CYFIP2. This first ultra-deep sequencing study highlighted genetic intra-tumor heterogeneity in pNEN, by the presence of lowabundance mutations. The importance of the ATRXIDAXX pathway was confirmed by the first-ever pNEN-specific protein-damaging hotspot mutation in DAXX. In this study, both novel genes, including the pro-apoptotic CYFIP2 gene and hedgehog signaling PTCH2, and novel pathways, such as the MAPK-ERK pathway, were implicated in pNEN.
\end{abstract}

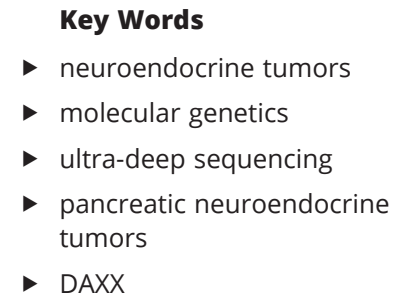

Endocrine-Related Cancer (2019) 26, 1-12 (c) 2019 Society for Endocrinology Published by Bioscientifica Ltd. Printed in Great Britain 


\section{Introduction}

Neuroendocrine neoplasms of the pancreas (pNENs), originating from the islet cells, are considered rare, although incidence is increasing (Dasari et al. 2017). pNENs can occur as part of genetic syndromes, such as multiple neuroendocrine neoplasia 1 (MEN1), VonHippel Lindau and tuberous sclerosis complex. However, most pNENs are sporadic tumors without familial history of NENs (Crona \& Skogseid 2016). Recently, non-familial pNENs have been genetically characterized using wholeexome and whole-genome sequencing in large cohorts of 40-102 patients (Jiao et al. 2011, Sadanandam et al. 2015, Scarpa et al. 2017). These studies identified MEN1 as most frequently mutated gene, in frequencies ranging from 37 to $44 \%$ of all sequenced tumors. Additionally, DAXX was found to be mutated in $22-25 \%$ of all tumor samples, while $A T R X$ was mutated in $10-17 \%$ of all tumors. Menin (the MEN1 protein), DAXX and ATRX are epigenetic regulators. Menin is involved in histone modification, while ATRX and DAXX play a role in alternative telomere lengthening and chromatin remodeling (Elsasser et al. 2011, Heaphy et al. 2011). Additionally, mutations in genes involved in the phosphoinositide-3-kinase/Akt/ mammalian target of rapamycin (PI3K/Akt/mTOR) pathway were found in $12-14 \%$ of tumors (Jiao et al. 2011, Scarpa et al. 2017). In the pivotal Radiant-3 trial, treatment with everolimus, an mTOR inhibitor, has demonstrated an improved progression-free survival in advanced pNENs (Yao et al. 2011). Hence, alterations in the PI3K/Akt/mTOR pathway, such as mutations in PTEN, could have clinical implications. However, no molecular predictive biomarker for everolimus treatment has yet been identified. Frequent tumor-specific copy number alterations in MEN1, ATRX, DAXX an PI3K/Akt/mTOR genes implicate these core pathways further (Scarpa et al. 2017). Recent efforts to describe molecular subtypes have led to the identification of five mutational signatures in pNEN, including the novel MUTYH signature (Scarpa et al. 2017). However, in more than $50 \%$ of all tumors, no dominant mutational signature could be identified (Scarpa et al. 2017). RNA expression analysis revealed three expression subtypes, respectively the insulinoma, MEN-1-like/intermediate and metastasis-like (MLP) subtype (Sadanandam et al. 2015, Scarpa et al. 2017). Clinical utility of these expression subtypes is subject of further study, as expression subtypes show a variable association with tumor grade, mainly in WHO 2010 grade 1 and 2 tumors (Sadanandam et al. 2015). Moreover, withinpatient and within-tumor heterogeneity in proliferation has been demonstrated in neuroendocrine neoplasm models and patients (Shi et al. 2015, Vandamme et al. $2015 a, 2016)$. In recent pNEN sequencing studies, average sequencing depth was 61- to 102-fold (Sadanandam et al. 2015, Scarpa et al. 2017). Although covering all the genome, these studies might lack sequencing power to reliably detect mutations present in a fraction of the cells, as these rare alleles might be present in less than 1 in 100 sequencing reads on a given genomic position (Gerstung et al. 2012). Therefore, current studies lack information on mutational heterogeneity. By increasing sequencing depth, mutations, present in a fraction of cells, can be reliably identified (Gerstung et al. 2012). This study is the first to use an ultra-deep targeted resequencing approach in pNENs to elucidate mutations, present in less than $10 \%$ of sequencing reads.

\section{Materials and methods}

\section{Sample collection and clinical data}

Patients diagnosed between 1997 and 2013 with a reported WHO 2010 grade 1 or 2 pNEN were retrospectively included in this study (Bosman et al. 2010). Patients with a familial syndrome were excluded. Formalin-fixed paraffinembedded (FFPE) samples of tumor and matched distant normal tissue, if available, of all patients was collected at the Erasmus Medical Center (Rotterdam, the Netherlands) and the Antwerp University Hospital (Antwerp, Belgium). All samples were reviewed by a dedicated pathologist for histology, Ki67 index, mitoses per high power field and tumor purity. Only samples with estimated tumor purity $>60 \%$ on hematoxylin and eosin-stained histological slide, after macrodissection, were included. Data on age, sex, TNM stage, age at diagnosis, secretion status, received treatments, disease-free survival and overall survival were collected. The study was approved by the Institutional Human Ethics Review Board of the Antwerp University Hospital. Informed consent was obtained from all patients for the use of excess tissue material for scientific research, based on the opt-out registry to document the objection of patients (as specified in Belgian and Dutch law).

\section{Targeted gene panel development}

A custom HaloPlex enrichment panel (Agilent Technologies) was developed to sequence all exons of 20 genes (Table 1). Genes were selected based upon patient and cell line sequencing results (Jiao et al. 2011, Vandamme et al. 2015b). PI3K/Akt/mTOR pathway-related Printed in Great Britain 


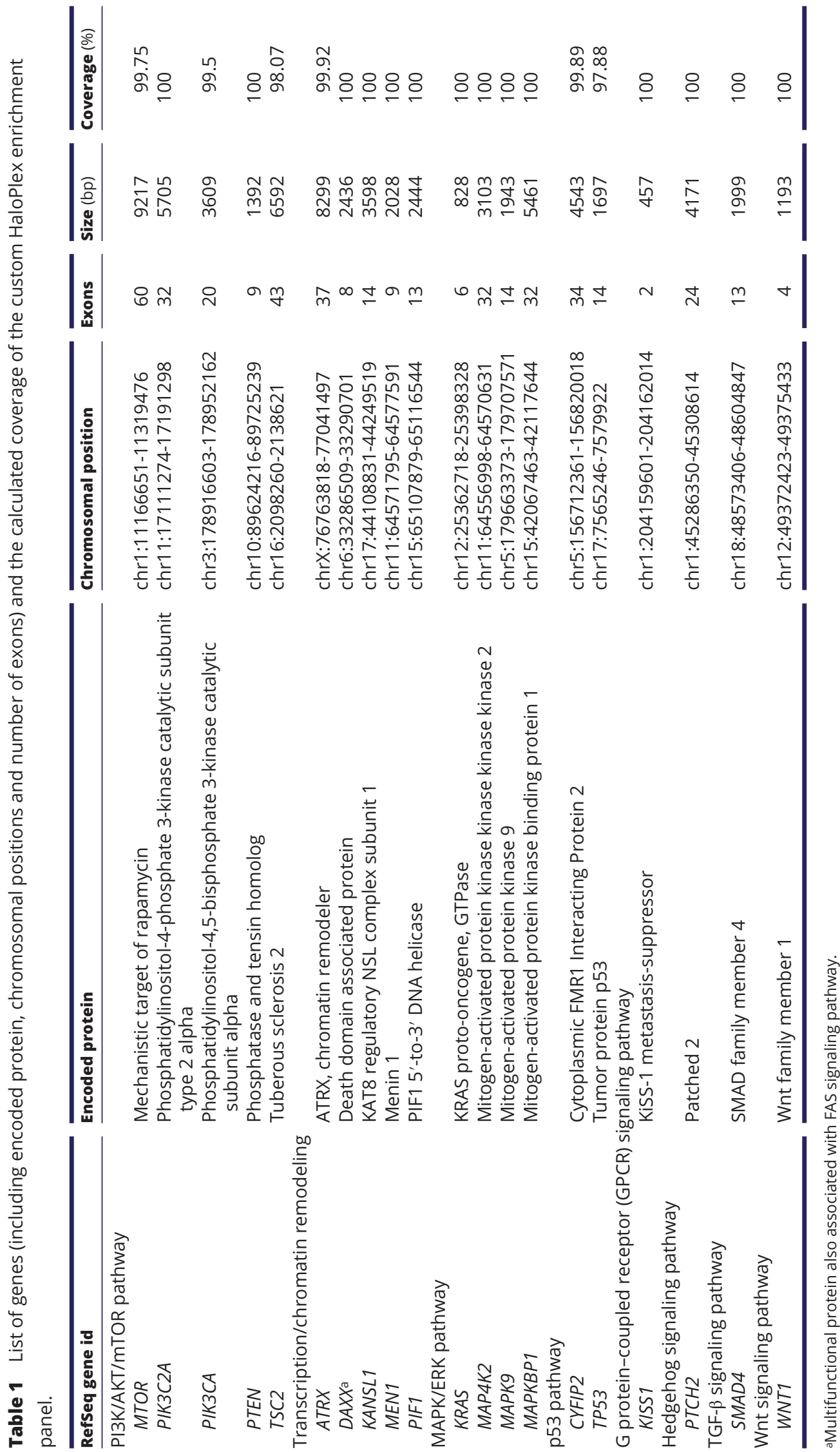


genes MTOR, PTEN, PIK3CA and TSC2 mutations have been found in pNEN patients and could have therapeutic implications (Jiao et al. 2011, Grabiner et al. 2014, Scarpa et al. 2017). The gene panel was extended with the PI3K-related PIK3C2A gene, as it was found mutated in pNEN cell line data (Vandamme et al. 2015b). Epigenetic modifiers could play a role in pNEN, as demonstrated by MEN1, ATRX and DAXX mutations identified previously in pNEN (Jiao et al. 2011, Scarpa et al. 2017). Next to these genes, KANSL1 and PIF1, two epigenetic modifiers found in pNEN cell line data, were included in the panel (Vandamme et al. 2015b). As preclinical data suggests a role for the MAPK/ERK pathway in pNEN, KRAS, MAP4K2, $M A P K 9$ and MAPKBP1 were added to the panel (Valentino et al. 2014, Vandamme et al. 2016). The SMAD4 gene has been found mutated in neuroendocrine neoplasms, both in patient material and in cell lines (Bartsch et al. 1999, Vandamme et al. 2015b). TP53 and CYFIP2 are two genes involved in apoptosis that are frequently implicated in oncogenesis. Both genes were found mutated in pNEN cell line data and were hence added to the panel (Vandamme et al. 2015b). Finally, the panel was extended with three genes (KISS1, PTCH2, WNT1) in known cancer-related pathways. All genes contained mutations in pNEN cell line data (Vandamme et al. 2015b). In our custom HaloPlex primer design, $99.59 \%$ of the 70,715 target kilobases of selected genes were covered by developed primers. The primer design was optimized for use with FFPE material and for sequencing on Illumina technology with a read length of 150 base pairs by increasing the number of amplicons to 19,838 .

\section{DNA isolation, HaloPlex enrichment and sequencing}

After macrodissection, ten slides of $5 \mu \mathrm{m}$ of both tumor and normal tissue were used as input for DNA isolation using the QIAamp DNA FFPE Tissue Kit (Qiagen), following manufacturer's instructions. The concentration of the isolated DNA was quantified using the Qubit 2.0 fluorometer with the dsDNA Broad Range Assay (Thermo Scientific). To determine the amount of input DNA for HaloPlex enrichment, quality and degradation of the DNA were checked following the FFPE-Derived DNA Quality Assessment-protocol (Agilent Technologies) using the LabChip GX (PerkinElmer) with a High-Sensitivity DNA kit (PerkinElmer). Next, all tumor samples and three representative normal samples were prepared for targeted resequencing using a custom HaloPlex Design enrichment, optimized for FFPE sample enrichment, following the FFPE-optimized protocol according to manufacturer's instructions. The enriched samples were hybridized, amplified and sequenced on two lanes of a paired-end flow cell using HiSeq 1500 (Illumina, San Diego, USA) platform in rapid run mode.

\section{Read alignment, variant calling and filtering}

Raw sequencing reads were analyzed using an in-house developed Perl-based workflow. First, FastQC software (version 1.0) was used to the assess quality of the raw data (Andrews 2010). Adapters and low-quality bases were trimmed using Cutadapt (version 1.2.1) and an in-house developed paired-end read quality trimmer (Vandeweyer et al. 2014), respectively. Paired-end reads were then aligned to the human reference genome (hg19, NCBI Build 37) using Burrows-Wheeler Aligner (BWA mem, version 0.7.3a) (Li \& Durbin 2009). Picard (version 1.88) was used to mark and remove duplicates. Afterward, the three aligned normals were merged using Samtools (version 0.1.18) (Li et al. 2009). After merging, somatic variant calling was performed on the tumor aligned data with VarScan2 (version 2.3.9) using the three merged normals as one merged normal (Koboldt et al. 2012). Alignments with mapping quality lower than 17 or nucleotides with base quality lower than 17 were ignored. The max perBAM depth was set on 30,000 avoiding excessive memory usage. No correction for tumor purity was set during the somatic calling, purities were kept on default (100\%). The variant calling files were first filtered using pyAmpli to eliminate false-positive variants introduced by ampliconbased enrichment (Beyens et al. 2017). All variants were annotated with ANNOVAR (Wang et al. 2010), and filtered using VariantDB (Vandeweyer et al. 2014) according to different criteria (Results section). Identified variants were validated in tumor and its matching normal tissue, if available, with Sanger sequencing on the 3130xl Genetic Analyzer (Applied Biosystems Inc.) platform and analyzed using CLC DNA Workbench v5 software (CLC Bio, Aarhus, Denmark). Data were visualized using the Maftools package (Mayakonda \& Koeffler 2016) in R (version 3.3.3). Survival statistics were generated using the survival and survminer package for R (version 3.3.3).

\section{Results}

\section{Patient characteristics}

Thirty-eight pNEN patients were included, of which 51\% were male. Of the 38 included patients, 13 patients had a functional tumor (11 insulinomas, one gastrinoma 
and one glucagonoma). Mean age at diagnosis was $53 \pm 14$ years. Of all patients, 9 (16\%) had metastatic disease at diagnosis (Supplementary Table S1, see section on supplementary data given at the end of this article). Tumor tissue was available for all included patients, while matched normal tissue was collected in 27 patients (71\%). Twenty-four patients were diagnosed with WHO grade 1 disease, while 13 patients had a WHO grade 2 tumor. Upon central pathology review, one patient was reclassified as a WHO 2010 grade 3 tumor, given the Ki67 index of $30 \%$. Median follow-up time was 6.3 years (range: $1.9-19.2$ years). Median overall survival was 13 years $(95 \%$ CI 11 years - not reached).

\section{Genetic alterations in pNENs}

Targeted resequencing of the selected HaloPlex enrichment 20-gene panel (see Materials and methods) resulted in a total of 416,711,794 reads, passing quality filtering, across 38 tumor samples and three normal samples. Of all reads, an average $80.4 \pm 5.0 \%$ mapped on target regions. Average target base coverage was $2663 \pm 1476$ and $94.7 \pm 1.9 \%$ of all target bases was covered at least 30 times. Using VarScan2, variants in the tumor were called against three merged normal samples. Afterward, the amplicon-based filtering method pyAmpli was deployed to withhold only alterations present in more than one amplicon (if more than one amplicon was in the enrichment design) (Beyens et al. 2017). Additionally, all variants that were marked as 'somatic' by VarScan2 were withheld. By this combined elimination, genetic alterations that are inherent to the enrichment with the HaloPlex panel and, hence, appear in all amplicons across all samples, are removed as false positive. Additionally, genetic alterations that are present in all three sequenced normal samples will most likely not be oncogenic driver mutations. Hence, these mutations can be discarded as common polymorphisms or artifacts when they are seen in the tumor samples. After this combined filtering, 17 indels and 2270 single nucleotide variations (SNVs) were withheld in the 38 tumors. The resulting median mutation burden of 0.80 per Megabase $(\mathrm{Mb}$, range $0.51-1.54)$ within the 20 selected genes, with a genomic length of $70.71 \mathrm{Mb}$, is in line with the previously reported genome-wide mutation burden of 0.82 per $\mathrm{Mb}$ in pNEN and is low in comparison to other tumor types (Scarpa et al. 2017). Although caution is needed when comparing mutation burden between the targeted sequencing panel and the previously reported whole-genome data, it seems that the 20 selected genes in the targeted panel show no enrichment for mutations in comparison to other genomic regions. Before further filtering, the median number of genetic alterations per tumor was 56 with the MTOR gene containing the most alterations (Fig. 1). The most frequent nucleotide substitutions were $\mathrm{C}<->\mathrm{T}$ transitions (Fig. 1). Across all samples, transitions made up $63.4 \% \pm 2.3 \%$ of the filtered SNVs, resulting in a ratio of transitions to transversions of $1.74 \pm 0.18$ in the presented gene panel. In human germline samples, an average transitions to transversion rate of 1.7 is usually seen genome-wide (Lynch 2010). A genome-wide mutation spectrum with a predominant $\mathrm{C}$ to $\mathrm{T} / \mathrm{G}$ to $\mathrm{A}$ transition pattern is seen in many adult cancers, including melanoma, breast, lung, colorectal, ovarian and pancreas adenocarcinoma (Greenman et al. 2007, Jones et al. 2008). However, in pNENs, a more even distribution of transversions and transitions has been reported previously, in line with the data in this study (Jiao et al. 2011).

\section{Identification and validation of somatic and germline mutations}

To identify mutations with possible functional impact, RefSeq synonymous as well as intronic SNVs and intronic indels were first removed from further analysis (Pruitt et al. 2014). To eliminate common SNPs, only variants with a minor allele frequency (MAF) smaller than 0.05 in the dbSNP v142, ESP65000 and 1000 Genomes databases were withheld (Sherry et al. 2001, Genomes Project et al. 2010). Final visual inspection of all remaining variants in Integrative Genomics Viewer (IGV version 2.2.5), led to the identification of 72 mutations with possible functional impact (Fig. 2) (Thorvaldsdottir et al. 2012). Of these 72 mutations, 42 alterations were identified in more than $10 \%$ of sequencing reads at that genomic position and were considered high-abundance alterations. These 42 alterations were Sanger sequenced in tumor tissue. Additionally, of 27 patients (71\%) corresponding normal tissue was available, allowing Sanger sequencing of normal tissue for 22 mutations. Out of the 42 highabundant variants, 35 were validated through Sanger sequencing, while 6 variants could not be detected in the Sanger electropherogram traces. For one variant, PCR amplification of the tumor DNA region containing the variant was unsuccessful, despite successful amplification in control DNA and use of different primers. Overall, $83.3 \%$ of all high-abundant variants could be validated (Supplementary Table S2). The 35 validated mutations contained three germline RefSeq non-synonymous variants, one in MAPKBP1 and two in PIF1 respectively, 
(
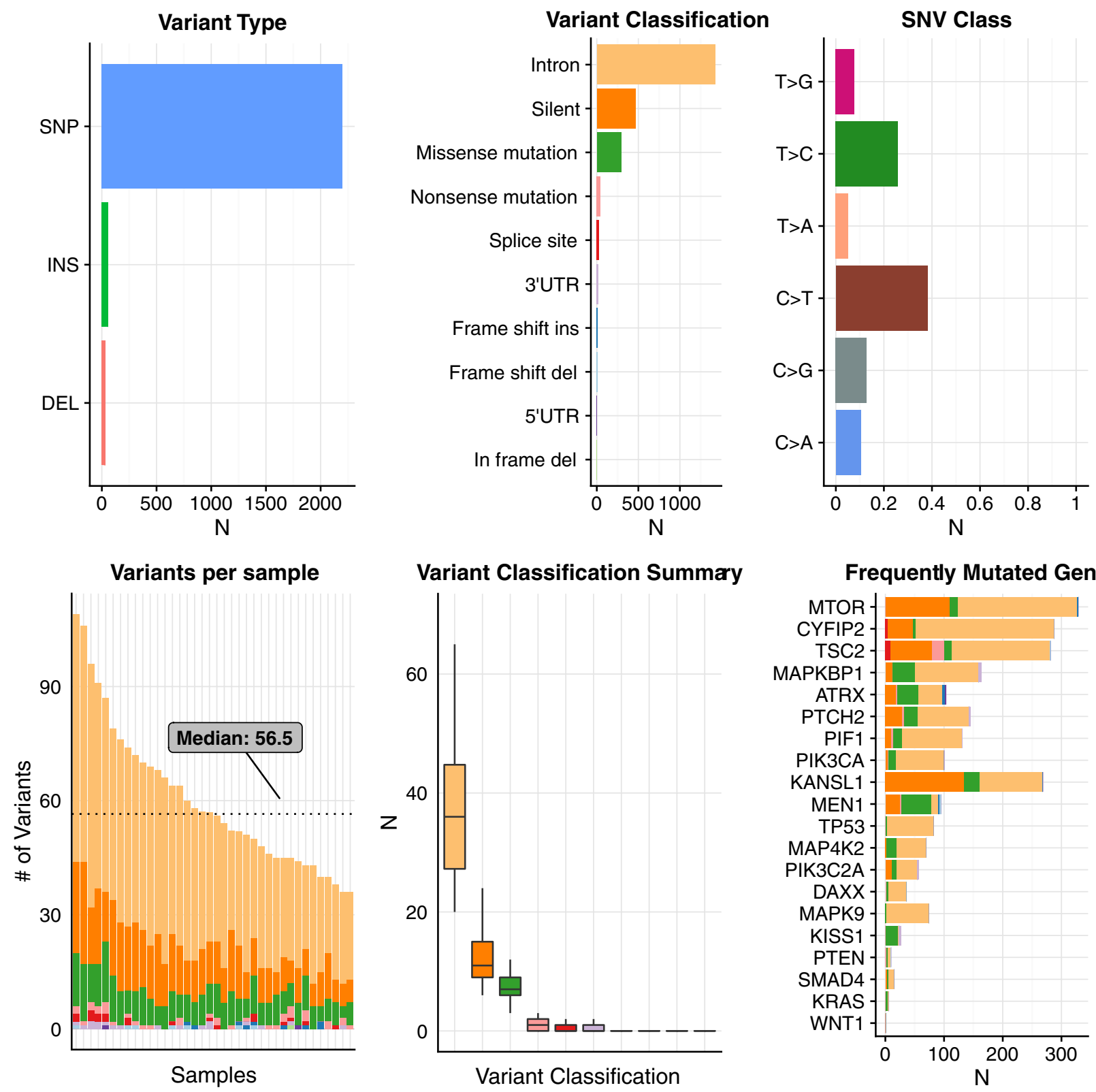

Frequently Mutated Genes
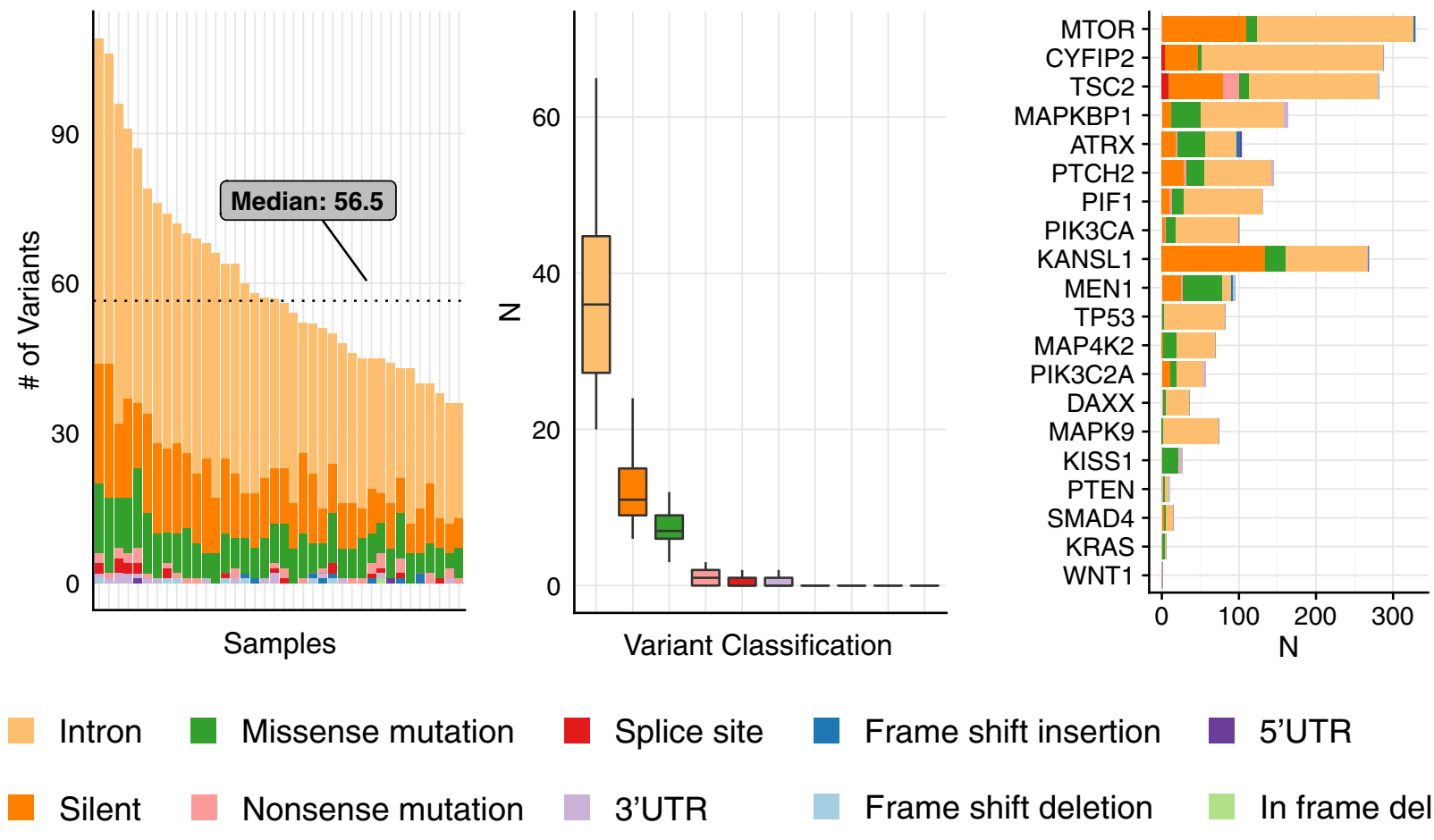

Splice site
3'UTR

Fram
shift insertion
5'UTR

Figure 1

Summary plot of SNVs $(N=2270)$, insertions $(N=9)$ and deletions $(N=8)$ of all 38 samples, after amplicon-based filtering.

all unique in three different tumors. None of the mutations were present in the cancer somatic mutation COSMIC v70 database (accession date: 12th April 2017), while two SNVs were reported in the dbSNP142 database with unknown clinical significance, respectively dbSNP142 rs139868280 (PIF1) and rs201725344 (MAPKBP1) (Sherry et al. 2001,
Forbes et al. 2017). The other PIF1 chr15:g.65108822C>T mutation was not found in any of the queried databases, including ExAc (version 03), 1000 Genomes Project (October 2014), dbSNP142 and COSMIC v70 (Sherry et al. 2001, Genomes Project et al. 2010, Lek et al. 2016, Forbes et al. 2017). 


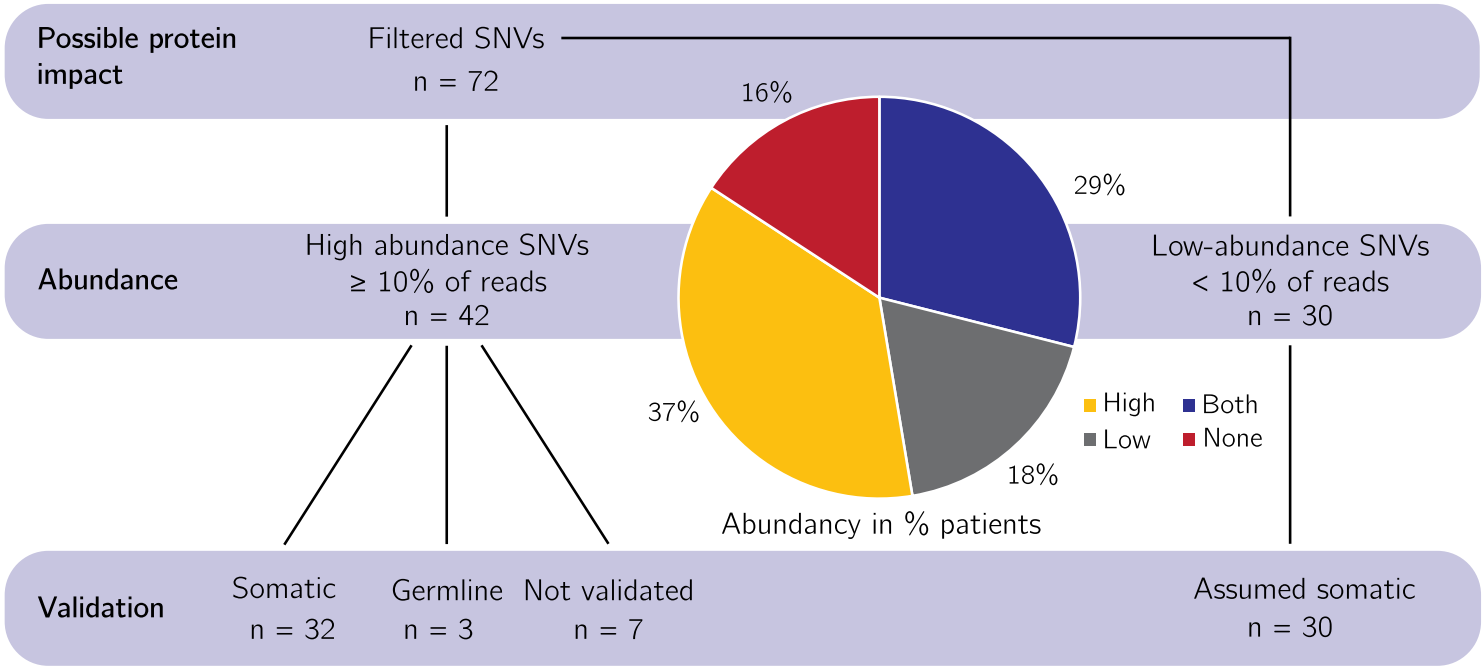

\section{Figure 2}

Filter strategy and abundancy of mutations across tumors. Filtered variants are SNVs or indels that are not RefSeq synonymous or intronic and have a minor allele frequency (MAF) $\leq 0.05$ in dbSNP v142, ESP65000 and 1000 Genomes. Abundance is considered low for alterations found in $<10 \%$ of targeted resequencing reads in tumor tissue and high when an alteration is present in $\geq 10 \%$ of targeted resequencing reads. Mutation are validated somatic when mutation is present in Sanger sequencing of tumor but not in normal (if available). Germline mutations are present in Sanger sequencing traces of both tumor and normal tissue. Not validated SNVs are those SNVs with absent Sanger traces or inconclusive results. All low-abundance SNVs or indels are assumed to be somatic. Pie-chart shows the percentage of patients whose tumors contained only low-abundance mutations (gray), only highabundance mutations (yellow), both (red) or no mutations (blue).

\section{Abundant mutations include a DAXX and CYFIP2 hotspot mutation}

Of the 32 validated high-abundant somatic mutations, 21 SNVs were annotated as non-synonymous by RefSeq, six as frameshift indels, three as stopgain SNVs, one as SNV in the 3'-UTR and one as nonframeshift indel. The most commonly mutated gene was DAXX with five high-abundance mutations, while four high-abundance mutations were found in MEN1, MAP4K2 and PTCH2. ATRX, KANSL1, TSC2 and MAPKB1 each contained three high-abundance mutations. These 8 genes accounted for $80 \%$ of all validated high-abundance mutations. No high-abundance mutations were found in PIF1, PIK3CA, PTEN, MAPK9, KRAS and TP53. Using variantDB, all variants were annotated for functional impact prediction using the MutationAssesor, MutationTaster, Provean and PolyPhen algorithms (Vandeweyer et al. 2014). Of all variants, $92.0 \%$ was predicted damaging by at least one algorithm, while $36.0 \%$ was predicted damaging by at least three algorithms. Three mutations were also seen in other tumor types, according to the COSMIC database (Forbes et al. 2017). Two recurrent, so-called hotspot, mutations were found, one in CYFIP2 and one in DAXX. The CYFIP2 non-synonymous variant g.156766140G $>\mathrm{A}$ (NM_001037333, p.D820N) was found in two pNEN tumors. This variant had previously been identified in skin squamous cell carcinoma, according to COSMIC, and was predicted to be damaging by MutationTaster and PROVEAN (Choi et al. 2012, Schwarz et al. 2014). Within the DAXX gene, one genomic position was altered differently in two tumors, yielding the non-synonymous g.33289247G>A (NM_001141970.1, p.S102L) and the stopgain g.33289247G>T (NM_001141970.1, p.S102X) mutations. Both mutations were predicted to be damaging by all used prediction algorithms, pointing toward a very likely $D A X X$ loss-of-function in these tumors. The non-synonymous g.33289247G $>\mathrm{A} D A X X$ mutations has previously been found as a somatic mutation in one lung squamous cell carcinoma, according to COSMIC.

\section{Low-abundance mutations contain a hotspot mutation in PTCH2}

Next to high-abundance mutations, 30 mutations were found in less than $10 \%$ of targeted resequencing reads in tumor tissue. These mutations are considered low-abundance mutations. As the detection limit of Sanger sequencing is around $10 \%$, these low-abundant alterations could not be Sanger sequenced (Tsiatis et al. 2010). However, the Sanger sequencing validation rate of $83 \%$ in the high-abundant mutations demonstrates that the used combined filtering strategy (see above) yields mainly true positives. Additionally, only 9\% of validated mutations was present in corresponding 
normal samples, illustrating that the employed filtering strategy selects for somatic variants. Hence, we assume that most of the low-abundance mutations are also both valid and somatic. RefSeq annotated 21 of these 30 lowabundance mutations as non-synonymous SNVs, four as frameshift indels, three as stopgain SNVs, one as splicing SNV and one as SNV in the 3'-UTR. The 3'-UTR PIK3C2A g.17111197_17111198del was found in three tumors. In addition, two intronic variants were found, the $D A X X$ g.33286734A>C which was seen in 8 pNENs, and the TSC2 g.2124481_2124482insG, identified in five tumors. However, functional impact of these intronic mutations remains unclear. When predicting protein impact on the 26 exonic SNVs with the MutationAssesor, MutationTaster, Provean and PolyPhen algorithms using variantDB, $84.0 \%$ of all SNVs was predicted damaging by at least one algorithm, while $52.0 \%$ was predicted to be damaging by three or more prediction algorithms (Vandeweyer et al. 2014). After inclusion of the four frameshift indels, which can be considered to have a deleterious impact on protein function, $86.6 \%$ of all mutations have a likely protein impact. Three mutations were previously found in cancer samples, according to the COSMIC database, including the non-synonymous TP53 g.7578457C $>\mathrm{T}$ mutation (NM_001276695.1, p.R158H), which was identified in 36 tumors of various origin (Forbes et al. 2017). Two tumors contained the same stopgain g.45292871C $>$ A mutation (NM_001166292, p.G828X) in PTCH2, predicted to be damaging by MutationTaster, PROVEAN and SIFT. This protein-damaging $\mathrm{PTCH} 2$ mutation is hence a novel lowabundance hotspot mutation within pNEN.

\section{Mutational signatures in pNEN}

In total, 32 tumors contained mutations while 6 tumors had no mutations that survived filtering (Fig. 3). Both low-abundance and high-abundance mutations were seen in 11 tumors (29\%). High-abundance mutations were seen exclusively in 14 tumors (37\%), while 7 tumors (18\%) only contained low-abundance mutations (Fig. 2). MEN1 was mutated most frequently in 8 out of 38 tumors (21\%). DAXX and ATRX were both mutated in 5 out of 38 tumors (13\%). The PI3K/Akt/mTOR-related gene TSC2 was mutated in 7 out of 38 tumors (18\%). Overall, mutations in PI3K/Akt/mTOR-related genes were seen in $29 \%$ of the tumors, including a validated non-synonymous g.1308007C $>$ T mutation in MTOR (NM_004958, p.A329T, rs35903812) not previously reported in pNEN. The mitogen-activated kinase and extracellular signal-regulated kinase (MAPK-ERK pathway) genes MAP4K2 and MAPKBP1 were each mutated in three tumors.

\section{Gene mutation plot after filtering}

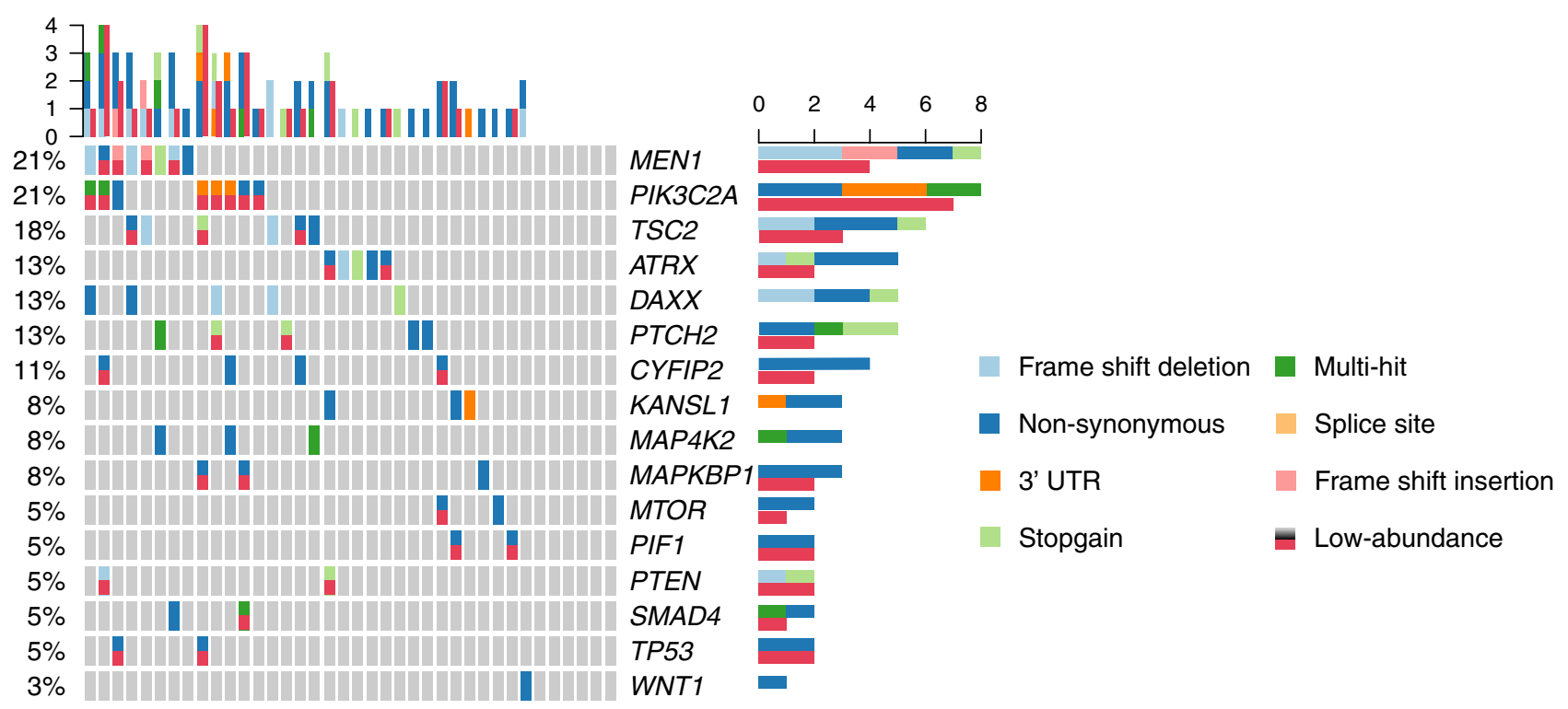

Figure 3

Mutation plot showing frequency, type of mutations, abundancy and mutational distribution of genetic alterations across 38 included tumors, after filtering and validation (if executed), including both high-abundance $(N=32)$ and low-abundance $(N=30)$ mutations. Low-abundance mutations are marked with red, in addition to color-coded mutation type. When a tumor contains multiple mutations within one gene, this is annotated as multi-hit (dark green).

https://erc.bioscientifica.com https://doi.org/10.1530/ERC-18-0120 (c) 2019 Society for Endocrinology Published by Bioscientifica Ltd. Printed in Great Britain 


\section{Discussion}

This study presents the first ultra-deep targeted resequencing of pNENs in archival tissue. The relative rarity of pNENs has led to only a limited number of largescale studies on the genetic constitution of pNENs (Jiao et al. 2011, Sadanandam et al. 2015, Scarpa et al. 2017). Additionally, these studies focus on a broad overview of frequent genetic alterations in pNEN and report highabundance mutations. These high-abundance mutations are present in the majority of tumors cells. However, various studies have demonstrated that the genetic make-up of primary tumors evolves dynamically in time (Stratton et al. 2009, Burrell et al. 2013). This timedependent change of the genetic alterations present in a tumor reflects the appearance and disappearance of subsets of tumor cells, so-called subclones, within one tumor (Stratton et al. 2009, Burrell et al. 2013). In our study, the use of ultra-deep sequencing allowed for the identification of genetic alterations that are present in a low fraction of the tumoral tissue, so-called low-abundance mutations. These low-abundance mutations are indicative for the genetic heterogeneity within a single tumor. Recent genetic studies have identified the insulinoma, MEN-1like/intermediate and metastasis-like (MLP) RNA expression subtype in pNENs (Sadanandam et al. 2015). Although the MEN-1-like tumors frequently contains MEN1 mutations, the mutational burden of highabundance mutations in other core pathways seems to be more variable across subtypes (Sadanandam et al. 2015). Additionally, DNA damage repair, chromatin modification, alternated telomere length and the PI3K/Akt/mTOR pathways have been highlighted as core altered pathways in pNEN (Jiao et al. 2011, Scarpa et al. 2017). In this study, we demonstrate that low-abundance mutations are found in these pathways. Hence, low-abundance mutations might help to better classify tumors. However, validation of these low-abundance mutations remains complex and its lack forms a limitation of our study. The gene with the most high-abundance mutations within our study was the tumor suppressor gene DAXX. ATRX and DAXX form a complex facilitating the incorporation of histone variant H3.3 at the telomeres and, consequently, play a role in alternative lengthening of telomeres (Lewis et al. 2010, Heaphy et al. 2011). In many cancer types, it has been demonstrated that alternative telomere lengthening (ATL) leads to a prolonged cell survival, which is a hallmark of cancer (Cesare \& Reddel 2010). In pNEN, ATL has been associated with a reduced disease-free survival (Singhi et al. 2017). Additionally, loss of ATRX and DAXX on Printed in Great Britain immunohistochemistry (IHC) staining, caused by inactivating mutations or copy number loss is associated with increased occurrence of metastasis and reduced disease-free survival (Scarpa et al. 2017, Singhi et al. 2017). Both high- and low-abundance mutations in DAXX and $A T R X$ were identified in this study, including the first-ever validated recurrent, hotspot, mutation in $D A X X$, with likely loss-of-function on protein level. In line with other studies, exonic (transcript coding or protein encoding) mutations in DAXX and ATRX were mutually exclusive (Jiao et al. 2011, Scarpa et al. 2017). As this is the first study to demonstrate the existence of low-abundance mutations in $A T R X$ and $D A X X$, conclusions on clinical impact are limited. Nonetheless, detection of low-abundance mutations might lead to the identification of DAXX and ATRX loss before this is apparent on IHC and, thus, an earlier identification of high-risk patients. Given the low number of events in the studied population (both in disease-recurrence and in mortality), this study is not adequately powered to evaluate prognostic relevance of these low-abundance mutations. Hence, further studies are needed. Menin, the nuclear protein encoded by MEN1, impacts ATL by negatively regulating hTERT (Lin \& Elledge 2003). Additionally, it plays a key role in chromatin remodeling and gene expression through histone acetylation and deacetylation (Kim et al. 2003). MEN1 mutations have been implicated in pNEN oncogenesis, both as part of the familial MEN1 syndrome, and in sporadic tumors (Corbo et al. 2010, Jiao et al. 2011, Crona \& Skogseid 2016, Scarpa et al. 2017). In 12-14\% of all pNEN patients, a mutation in PI3K/Akt/mTOR-related genes is reported, including mutations in PTEN, MTOR, DEPDC5, TSC1, TSC2 and PIK3CA (Jiao et al. 2011, Chou et al. 2016, Scarpa et al. 2017). In this study, PI3K/Akt/ mTOR-related mutations could be identified in $29 \%$ of all tumors, including a novel p.A329T mutation in MTOR in pNEN. This increased frequency of mutations in the PI3K/ Akt/mTOR-related genes is due to the detection of lowabundance mutations in these tumor samples. The relation of everolimus efficacy and PI3K/Akt/mTOR pathwayrelated mutations remains the subject of study in pNEN. However, in other cancer types, mutations of the PI3K/ Akt/mTOR pathways seem to confer everolimus sensitivity (Grabiner et al. 2014, Wagle et al. 2014). In neuroendocrine neoplasms and other cancer types, cross-talk activation between the PI3K/Akt/mTOR pathway and the mitogen-activated kinase and extracellular signal-regulated kinase (MAPK-ERK pathway) through PI3K has been described (Carracedo et al. 2008, Zitzmann et al. 2010, Valentino et al. 2014). Until now, no mutations 
in MAPK-ERK pathway-related genes have been reported in pNEN. In this study, mutations in MAP4K2 and $M A P K B P 1$ were identified, further implicating this pathway in pNEN and providing additional rationale for the use of MEK-inhibitors in pNEN, either in combination with mTOR inhibitors or alone. Although TP53 is frequently mutated in other cancers, TP53 mutation frequency is low in pNEN, as demonstrated by only two low-abundance TP53 mutations being present in our cohort (Jiao et al. 2011, Scarpa et al. 2017). Additionally, the pro-apoptotic gene CYFIP2 was mutated in four tumors. In two tumors, the exact same mutation was present, a so-called hotspot mutation. This validated mutation has never been reported before in pNEN and is predicted to be protein-damaging, warranting further studies into the role of CYFIP2 in pNEN. Three mutations (one high-abundance and two lowabundance) were found in SMAD4. Unlike in small intestinal neuroendocrine neoplasms, where SMAD4 loss is relatively common, the frequency of SMAD4 mutations in pNEN has been the matter of debate (Bartsch et al. 1999, Perren et al. 2003, Banck et al. 2013). Multiple lowabundance recurrent mutations were identified, including a hotspot mutation in PTCH2. PTCH2 encodes the Patched 2 protein, which is involved in hedgehog signaling (Smyth et al. 1999, Rahnama et al. 2004). PTCH2 mutations haven been found in basal cell carcinoma, medulloblastoma and rhabdomyosarcoma and myeloproliferative neoplasms (Smyth et al. 1999, Klein et al. 2016, Taeubner et al. 2018). However, this is the first time that hedgehog signaling has been implicated in pNENs. As the technical limitations of Sanger sequencing did not allow validation of this lowabundance mutation, further studies in replication cohorts are needed to confirm hedgehog signaling as a novel pathway in pNENs. Given that various hedgehog signaling inhibitors have been approved for use in basal cell carcinoma, confirmatory studies on the role of hedgehog signaling could open new therapeutic options for pNENs, harboring PTCH2 mutations (Lacouture et al. 2016). Finally, the limited availability of fresh-frozen tissue in pNEN could hinder the implementation of next-generation sequencing technology in pNEN diagnostics. In contrast, archival tissue, necessary for histological diagnosis of pNEN, is frequently available and can be easily manipulated and stored in a cost-effective manner. A possible limitation of the use of FFPE is the induction of false-positive mutations by formalin fixation (Williams et al. 1999). These mutations often follow a deamination pattern, resulting in transitions $(\mathrm{C}>\mathrm{T}$ or $\mathrm{G}>\mathrm{A})$. Hence, when formalin fixation would result in a considerable number of additional induced mutations, there would be an increase in the number of transitions $(\mathrm{C}>\mathrm{T}$ or $\mathrm{G}>\mathrm{A})$ in comparison to transversions $(A>C$ or $G>T)$. However, the ratio of transitions to transversion in this study is similar to transition-transversion ratios seen in normal human (germline) samples and in pNEN samples from freshfrozen tissue (Jiao et al. 2011). Therefore, the impact of formalin fixation can be considered to be relatively limited. To further reduce any potential impact, the in-house developed pyAmpli tool filters specifically for mutations present in more than one amplicon and, thus, amplified DNA fragment (Beyens et al. 2017). As fixation-induced 'deamination' is stochastic and happens randomly in all DNA strands, the chance that two different DNA molecules have exactly the same formalin fixation induced is very limited. Hence, our study demonstrates that by using this approach archival FFPE tissue can be used reliably in genetic analysis of pNENs.

In conclusion, this study adds to the growing body of evidence on the broad genetic constitution of pNENs by demonstrating the presence of low-abundance mutations and genetic heterogeneity in pNENs. We highlight the importance of the $A T R X / D A X X$ pathway by reporting the first-ever pNEN-specific protein-damaging hotspot mutation in $D A X X$, and uncover novel genes and pathways involved in pNENs, including pro-apoptotic CYFIP2, hedgehog signaling and the MAPK-ERK pathway.

\section{Supplementary data}

This is linked to the online version of the paper at https://doi.org/10.1530/ ERC-18-0120.

\section{Declaration of interest}

Timon Vandamme: advisory role and speakers' fees for Ipsen and Novartis. Wouter de Herder: advisory role and speakers' fees for Ipsen and Novartis. Marc Peeters: advisory role and speakers' fees for Ipsen and Novartis. Matthias Beyens, Ken Op de Beeck, Fadime Dogan, Peter M van Koetsveld, Patrick Pauwels, Guy Van Camp, Leo J Hofland, Gitta Boons, Anne Schepers, Kimberley Kamp, Katharina Biermann: No conflicts of interest to declare.

\section{Funding}

This work was supported by the Flemish Agency of Scientifc Research (FWO grant G.0327.13N) and the ENETS-Ipsen 2013 Translational Research Fellowship.

\section{Author contribution statement}

$T V, M B, G B, A$ S performed the sequencing experiments. T V, M B, $\mathrm{KO}$ d B processed the experimental data, performed the analysis, drafted the manuscript and designed the figures. P P, K B and LJ H performed pathological review of all tumor and normal samples and aided in patient selection. $\mathrm{K} \mathrm{K}$ and $\mathrm{W} W \mathrm{~W} \mathrm{H}$ aided in patient selection and collected clinicopathogical data. L J H, W W D H, M P and G V C were involved in 
planning and supervised the work. All authors discussed the results and commented on the manuscript.

\section{Acknowledgements}

The authors would like to thank Lesley De Backer from the Multidisciplinair Oncologisch Centrum Antwerpen and NETwerk for her support in collecting clinicopathological data of all patients included at the University Hospital Antwerp.

\section{References}

Andrews S 2010 FastQC: a quality control tool for high throughput sequence data. Cambridge, UK: Babraham Institute. (available at: http://www.bioinformatics.babraham.ac.uk/projects/fastqc)

Banck MS, Kanwar R, Kulkarni AA, Boora GK, Metge F, Kipp BR, Zhang L, Thorland EC, Minn KT, Tentu R, et al. 2013 The genomic landscape of small intestine neuroendocrine tumors. Journal of Clinical Investigation 123 2502-2508. (https://doi.org/10.1172/JCI67963)

Bartsch D, Hahn SA, Danichevski KD, Ramaswamy A, Bastian D, Galehdari H, Barth P, Schmiegel W, Simon B \& Rothmund M 1999 Mutations of the DPC4/Smad4 gene in neuroendocrine pancreatic tumors. Oncogene 18 2367-2371. (https://doi.org/10.1038/sj. onc.1202585)

Beyens M, Boeckx N, Van Camp G, Op de Beeck K \& Vandeweyer G 2017 pyAmpli: an amplicon-based variant filter pipeline for targeted resequencing data. BMC Bioinformatics 18 554. (https://doi. org/10.1186/s12859-017-1985-1)

Bosman FT, Carneiro F, Hruban RH \& Theise ND 2010 WHO Classification of Tumours of the Digestive System. Lyon: IARC Press.

Burrell RA, McGranahan N, Bartek J \& Swanton C 2013 The causes and consequences of genetic heterogeneity in cancer evolution. Nature 501 338-345. (https://doi.org/10.1038/nature12625)

Carracedo A, Ma L, Teruya-Feldstein J, Rojo F, Salmena L, Alimonti A, Egia A, Sasaki AT, Thomas G, Kozma SC, et al. 2008 Inhibition of mTORC1 leads to MAPK pathway activation through a PI3Kdependent feedback loop in human cancer. Journal of Clinical Investigation 118 3065-3074. (https://doi.org/10.1172/JCI34739)

Cesare AJ \& Reddel RR 2010 Alternative lengthening of telomeres: models, mechanisms and implications. Nature Reviews Genetics $\mathbf{1 1}$ 319-330. (https://doi.org/10.1038/nrg2763)

Choi Y, Sims GE, Murphy S, Miller JR \& Chan AP 2012 Predicting the functional effect of amino acid substitutions and indels. PLOS ONE 7 e46688. (https://doi.org/10.1371/journal.pone.0046688)

Chou WC, Lin PH, Yeh YC, Shyr YM, Fang WL, Wang SE, Liu CY, Chang PM, Chen MH, Hung YP, et al. 2016 Genes involved in angiogenesis and mTOR pathways are frequently mutated in Asian patients with pancreatic neuroendocrine tumors. International Journal of Biological Sciences 12 1523-1532. (https://doi.org/10.7150/ijbs.16233)

Corbo V, Dalai I, Scardoni M, Barbi S, Beghelli S, Bersani S, Albarello L, Doglioni C, Schott C, Capelli P, et al. 2010 MEN1 in pancreatic endocrine tumors: analysis of gene and protein status in 169 sporadic neoplasms reveals alterations in the vast majority of cases. EndocrineRelated Cancer 17 771-783. (https://doi.org/10.1677/ERC-10-0028)

Crona J \& Skogseid B 2016 GEP- NETS UPDATE: genetics of neuroendocrine tumors. European Journal of Endocrinology $\mathbf{1 7 4}$ R275-R290. (https://doi.org/10.1530/EJE-15-0972)

Dasari A, Shen C, Halperin D, Zhao B, Zhou S, Xu Y, Shih T \& Yao JC 2017 Trends in the incidence, prevalence, and survival outcomes in patients with neuroendocrine tumors in the United States. JAMA Oncology 3 1335-1342. (https://doi.org/10.1001/ jamaoncol.2017.0589)

Elsasser SJ, Allis CD \& Lewis PW 2011 Cancer. New epigenetic drivers of cancers. Science 331 1145-1146. (https://doi.org/10.1126/ science.1203280)
Forbes SA, Beare D, Boutselakis H, Bamford S, Bindal N, Tate J, Cole CG, Ward S, Dawson E, Ponting L, et al. 2017 COSMIC: somatic cancer genetics at high-resolution. Nucleic Acids Research 45 D777-D783. (https://doi.org/10.1093/nar/gkw1121)

Genomes Project C, Abecasis GR, Altshuler D, Auton A, Brooks LD, Durbin RM, Gibbs RA, Hurles ME \& McVean GA 2010 A map of human genome variation from population-scale sequencing. Nature 467 1061-1073. (https://doi.org/10.1038/nature09534)

Gerstung M, Beisel C, Rechsteiner M, Wild P, Schraml P, Moch H \& Beerenwinkel N 2012 Reliable detection of subclonal singlenucleotide variants in tumour cell populations. Nature Communications 3 811. (https://doi.org/10.1038/ncomms1814)

Grabiner BC, Nardi V, Birsoy K, Possemato R, Shen K, Sinha S, Jordan A, Beck AH \& Sabatini DM 2014 A diverse array of cancer-associated MTOR mutations are hyperactivating and can predict rapamycin sensitivity. Cancer Discovery 4 554-563. (https://doi. org/10.1158/2159-8290.CD-13-0929)

Greenman C, Stephens P, Smith R, Dalgliesh GL, Hunter C, Bignell G, Davies H, Teague J, Butler A, Stevens C, et al. 2007 Patterns of somatic mutation in human cancer genomes. Nature 446 153-158. (https://doi.org/10.1038/nature05610)

Heaphy CM, de Wilde RF, Jiao Y, Klein AP, Edil BH, Shi C, Bettegowda C, Rodriguez FJ, Eberhart CG, Hebbar S, et al. 2011 Altered telomeres in tumors with ATRX and DAXX mutations. Science 333 425. (https:// doi.org/10.1126/science.1207313)

Jiao Y, Shi C, Edil BH, de Wilde RF, Klimstra DS, Maitra A, Schulick RD, Tang LH, Wolfgang CL, Choti MA, et al. 2011 DAXX/ATRX, MEN1, and mTOR pathway genes are frequently altered in pancreatic neuroendocrine tumors. Science 331 1199-1203. (https://doi. org/10.1126/science.1200609)

Jones S, Zhang X, Parsons DW, Lin JC, Leary RJ, Angenendt P, Mankoo P, Carter H, Kamiyama H, Jimeno A, et al. 2008 Core signaling pathways in human pancreatic cancers revealed by global genomic analyses. Science 321 1801-1806. (https://doi.org/10.1126/science.1164368)

Kim H, Lee JE, Cho EJ, Liu JO \& Youn HD 2003 Menin, a tumor suppressor, represses JunD-mediated transcriptional activity by association with an mSin3A-histone deacetylase complex. Cancer Research 63 6135-6139.

Klein C, Zwick A, Kissel S, Forster CU, Pfeifer D, Follo M, Illert AL, Decker S, Benkler T, Pahl H, et al. 2016 Ptch2 loss drives myeloproliferation and myeloproliferative neoplasm progression. Journal of Experimental Medicine 213 273-290. (https://doi. org/10.1084/jem.20150556)

Koboldt DC, Zhang Q, Larson DE, Shen D, McLellan MD, Lin L, Miller CA, Mardis ER, Ding L \& Wilson RK 2012 VarScan 2: somatic mutation and copy number alteration discovery in cancer by exome sequencing. Genome Research 22 568-576. (https://doi.org/10.1101/gr.129684.111)

Lacouture ME, Dreno B, Ascierto PA, Dummer R, Basset-Seguin N, Fife K, Ernst S, Licitra L, Neves RI, Peris K, et al. 2016 Characterization and management of hedgehog pathway inhibitor-related adverse events in patients with advanced basal cell carcinoma. Oncologist 21 1218-1229. (https://doi.org/10.1634/theoncologist.2016-0186)

Lek M, Karczewski KJ, Minikel EV, Samocha KE, Banks E, Fennell T, O'Donnell-Luria AH, Ware JS, Hill AJ, Cummings BB, et al. 2016 Analysis of protein-coding genetic variation in 60,706 humans. Nature 536 285-291. (https://doi.org/10.1038/nature19057)

Lewis PW, Elsaesser SJ, Noh KM, Stadler SC \& Allis CD 2010 Daxx is an H3.3-specific histone chaperone and cooperates with ATRX in replication-independent chromatin assembly at telomeres. PNAS $\mathbf{1 0 7}$ 14075-14080. (https://doi.org/10.1073/pnas.1008850107)

Li H \& Durbin R 2009 Fast and accurate short read alignment with Burrows-Wheeler transform. Bioinformatics 25 1754-1760. (https:// doi.org/10.1093/bioinformatics/btp324)

Li H, Handsaker B, Wysoker A, Fennell T, Ruan J, Homer N, Marth G, Abecasis G, Durbin R \& Genome Project Data Processing S 2009 The sequence alignment/map format and SAMtools. Bioinformatics $\mathbf{2 5}$ 2078-2079. (https://doi.org/10.1093/bioinformatics/btp352) 
Lin SY \& Elledge SJ 2003 Multiple tumor suppressor pathways negatively regulate telomerase. Cell 113 881-889. (https://doi.org/10.1016/ S0092-8674(03)00430-6)

Lynch M 2010 Rate, molecular spectrum, and consequences of human mutation. PNAS 107 961-968. (https://doi.org/10.1073/ pnas.0912629107)

Mayakonda A \& Koeffler P 2016 Maftools: efficient analysis, visualization and summarization of MAF files from large-scale cohort based cancer studies. BioRxiv. (https://doi.org/doi.org/10.1101/052662)

Perren A, Saremaslani P, Schmid S, Bonvin C, Locher T, Roth J, Heitz PU \& Komminoth P 2003 DPC4/Smad4: no mutations, rare allelic imbalances, and retained protein expression in pancreatic endocrine tumors. Diagnostic Molecular Pathology 12 181-186. (https://doi. org/10.1097/00019606-200312000-00001)

Pruitt KD, Brown GR, Hiatt SM, Thibaud-Nissen F, Astashyn A, Ermolaeva O, Farrell CM, Hart J, Landrum MJ, McGarvey KM, et al. 2014 RefSeq: an update on mammalian reference sequences. Nucleic Acids Research 42 D756-D763. (https://doi.org/10.1093/nar/gkt1114)

Rahnama F, Toftgard R \& Zaphiropoulos PG 2004 Distinct roles of PTCH2 splice variants in Hedgehog signalling. Biochemical Journal 378 325-334. (https://doi.org/10.1042/bj20031200)

Sadanandam A, Wullschleger S, Lyssiotis CA, Grotzinger C, Barbi S, Bersani S, Korner J, Wafy I, Mafficini A, Lawlor RT, et al. 2015 A cross-species analysis in pancreatic neuroendocrine tumors reveals molecular subtypes with distinctive clinical, metastatic, developmental, and metabolic characteristics. Cancer Discovery 5 1296-1313. (https://doi.org/10.1158/2159-8290.CD-15-0068)

Scarpa A, Chang DK, Nones K, Corbo V, Patch AM, Bailey P, Lawlor RT, Johns AL, Miller DK, Mafficini A, et al. 2017 Whole-genome landscape of pancreatic neuroendocrine tumours. Nature 543 65-71. (https://doi.org/10.1038/nature21063)

Schwarz JM, Cooper DN, Schuelke M \& Seelow D 2014 MutationTaster2: mutation prediction for the deep-sequencing age. Nature Methods $\mathbf{1 1}$ 361-362. (https://doi.org/10.1038/nmeth.2890)

Sherry ST, Ward MH, Kholodov M, Baker J, Phan L, Smigielski EM \& Sirotkin K 2001 dbSNP: the NCBI database of genetic variation. Nucleic Acids Research 29 308-311. (https://doi.org/10.1093/nar/29.1.308)

Shi C, Gonzalez RS, Zhao Z, Koyama T, Cornish TC, Hande KR, Walker R, Sandler M, Berlin J \& Liu EH 2015 Liver metastases of small intestine neuroendocrine tumors: Ki-67 heterogeneity and World Health Organization grade discordance with primary tumors. American Journal of Clinical Pathology 143 398-404. (https://doi. org/10.1309/AJCPQ55SKOCYFZHN)

Singhi AD, Liu TC, Roncaioli JL, Cao D, Zeh HJ, Zureikat AH, Tsung A, Marsh JW, Lee KK, Hogg ME, et al. 2017 Alternative lengthening of telomeres and loss of DAXX/ATRX expression predicts metastatic disease and poor survival in patients with pancreatic neuroendocrine tumors. Clinical Cancer Research 23 600-609. (https://doi. org/10.1158/1078-0432.CCR-16-1113)

Smyth I, Narang MA, Evans T, Heimann C, Nakamura Y, ChenevixTrench G, Pietsch T, Wicking C \& Wainwright BJ 1999 Isolation and characterization of human patched 2 (PTCH2), a putative tumour suppressor gene inbasal cell carcinoma and medulloblastoma on chromosome 1p32. Human Molecular Genetics 8 291-297. (https:// doi.org/10.1093/hmg/8.2.291)

Stratton MR, Campbell PJ \& Futreal PA 2009 The cancer genome. Nature 458 719-724. (https://doi.org/10.1038/nature07943)

Taeubner J, Brozou T, Qin N, Bartl J, Ginzel S, Schaper J, Felsberg J, Fulda S, Vokuhl C, Borkhardt A, et al. 2018 Congenital embryonal rhabdomyosarcoma caused by heterozygous concomitant PTCH1 and PTCH2 germline mutations. European Journal of Human Genetics 26 137-142. (https://doi.org/10.1038/s41431-017-0048-4)

Thorvaldsdottir H, Robinson JT \& Mesirov JP 2012 Integrative genomics viewer (IGV): high-performance genomics data visualization and exploration. Briefings in Bioinformatics 14 178-192. (https://doi. org/10.1093/bib/bbs017)

Tsiatis AC, Norris-Kirby A, Rich RG, Hafez MJ, Gocke CD, Eshleman JR \& Murphy KM 2010 Comparison of Sanger sequencing, pyrosequencing, and melting curve analysis for the detection of KRAS mutations: diagnostic and clinical implications. Journal of Molecular Diagnostics 12 425-432. (https://doi.org/10.2353/jmoldx.2010.090188)

Valentino JD, Li J, Zaytseva YY, Mustain WC, Elliott VA, Kim JT, Harris JW, Campbell K, Weiss HL, Wang C, et al. 2014 Co-targeting the PI3K and RAS pathways for the treatment of neuroendocrine tumors. Clinical Cancer Research 20 1212-1222. (https://doi. org/10.1158/1078-0432.CCR-13-1897)

Vandeweyer G, Van Laer L, Loeys B, Van den Bulcke T \& Kooy RF 2014 VariantDB: a flexible annotation and filtering portal for next generation sequencing data. Genome Medicine 6 74. (https://doi. org/10.1186/s13073-014-0074-6)

Vandamme T, Beyens M, Peeters M, Van Camp G \& Op de Beeck K $2015 a$ Next generation exome sequencing of pancreatic neuroendocrine tumor cell lines BON-1 and QGP-1 reveals different lineages. Cancer Genetics 208 523-523. (https://doi.org/10.1016/j. cancergen.2015.07.003)

Vandamme T, Peeters M, Dogan F, Pauwels P, Van Assche E, Beyens M, Mortier G, Vandeweyer G, de Herder W, Van Camp G, et al. 2015b Whole-exome characterization of pancreatic neuroendocrine tumor cell lines BON-1 and QGP-1. Journal of Molecular Endocrinology $\mathbf{5 4}$ 137-147. (https://doi.org/10.1530/JME-14-0304)

Vandamme T, Beyens M, de Beeck KO, Dogan F, van Koetsveld PM, Pauwels P, Mortier G, Vangestel C, de Herder W, Van Camp G, et al. 2016 Long-term acquired everolimus resistance in pancreatic neuroendocrine tumours can be overcome with novel PI3K-AKTmTOR inhibitors. British Journal of Cancer 114 650-658. (https://doi. org/10.1038/bjc.2016.25)

Wagle N, Grabiner BC, Van Allen EM, Amin-Mansour A, TaylorWeiner A, Rosenberg M, Gray N, Barletta JA, Guo Y, Swanson SJ, et al. 2014 Response and acquired resistance to everolimus in anaplastic thyroid cancer. New England Journal of Medicine 371 1426-1433. (https://doi.org/10.1056/NEJMoa1403352)

Wang K, Li M \& Hakonarson H 2010 ANNOVAR: functional annotation of genetic variants from high-throughput sequencing data. Nucleic Acids Research 38 e164. (https://doi.org/10.1093/nar/ gkq603)

Williams C, Ponten F, Moberg C, Soderkvist P, Uhlen M, Ponten J, Sitbon G \& Lundeberg J 1999 A high frequency of sequence alterations is due to formalin fixation of archival specimens American Journal of Pathology 155 1467-1471. (https://doi. org/10.1016/S0002-9440(10)65461-2)

Yao JC, Shah MH, Ito T, Bohas CL, Wolin EM, Van Cutsem E, Hobday TJ, Okusaka T, Capdevila J, de Vries EG, et al. 2011 Everolimus for advanced pancreatic neuroendocrine tumors. New England Journal of Medicine 364 514-523. (https://doi.org/10.1056/ NEJMoa1009290)

Zitzmann K, Ruden J, Brand S, Goke B, Lichtl J, Spottl G \& Auernhammer CJ 2010 Compensatory activation of Akt in response to mTOR and Raf inhibitors - a rationale for dual-targeted therapy approaches in neuroendocrine tumor disease. Cancer Letters 295 100-109. (https://doi.org/10.1016/j.canlet.2010.02.018)

Received in final form 16 July 2018

Accepted 18 July 2018

Accepted Preprint published online 18 July 2018 https://erc.bioscientifica.com https://doi.org/10.1530/ERC-18-0120
(C) 2019 Society for Endocrinology Published by Bioscientifica Ltd. Printed in Great Britain 